\title{
QUANTITATIVE LINEARIZED STUDY OF THE BOLTZMANN COLLISION OPERATOR AND APPLICATIONS*
}

\author{
CLÉMENT MOUHOT ${ }^{\dagger}$
}

\begin{abstract}
We present recent results [4, 28, 29] about the quantitative study of the linearized Boltzmann collision operator, and its application to the study of the trend to equilibrium for the spatially homogeneous Boltzmann equation for hard spheres.
\end{abstract}

Key words. Boltzmann equation, spatially homogeneous, linearized Boltzmann collision operator, spectrum, spectral gap, explicit, trend to equilibrium, rate of convergence.

AMS subject classifications. 76P05, 82B40, 82C40, 82D05.

\section{Introduction}

In this paper, we present some of our recent works [4, 28, 29] (the first one [4] being in collaboration with Céline Baranger) on the quantitative study of the linearized Boltzmann collision operator and its application to the quantitative study of trend to equilibrium. This first section shall be devoted to the introduction of the Boltzmann equation and the motivation of this study. Section 2 recalls classical results on the linearized Boltzmann collision operator, and Section 3 and 4 present some new explicit estimates on this operator. Finally Section 5 presents an application of these explicit estimates in the study of explicit rate of convergence to equilibrium for the (nonlinear) spatially homogeneous Boltzmann equation.

1.1. The Boltzmann equation. The Boltzmann equation describes the behavior of a dilute gas when the only interactions taken into account are binary collisions, by means of an evolution equation on the time-dependent particle distribution function in the phase space. In the case where this distribution function is assumed to be independent of the position, we obtain the spatially homogeneous Boltzmann equation:

$$
\frac{\partial f}{\partial t}=Q(f, f), \quad v \in \mathbb{R}^{N}, \quad t \geq 0
$$

in dimension $N \geq 2$. In spite of the strong restriction that this assumption of spatial homogeneity constitutes, it has proven an interesting and inspiring case for studying qualitative properties of the Boltzmann equation. In equation (1.1), $Q$ is the quadratic Boltzmann collision operator, defined by the bilinear form

$$
Q(g, f)=\int_{\mathbb{R}^{N} \times \mathbb{S}^{N-1}} B\left(g_{*}^{\prime} f^{\prime}-g_{*} f\right) d v_{*} d \sigma .
$$

Here we have used the shorthands $f^{\prime}=f\left(v^{\prime}\right), g_{*}=g\left(v_{*}\right)$ and $g_{*}^{\prime}=g\left(v_{*}^{\prime}\right)$, where

$$
v^{\prime}=\frac{v+v_{*}}{2}+\frac{\left|v-v_{*}\right|}{2} \sigma, \quad v_{*}^{\prime}=\frac{v+v_{*}}{2}-\frac{\left|v-v_{*}\right|}{2} \sigma
$$

stand for the pre-collisional velocities of particles which after collision have velocities $v$ and $v_{*} . B$ is the Boltzmann collision kernel determined by physics (related to the

\footnotetext{
* Support by the European network HYKE, funded by the EC as contract HPRN-CT-2002-00282, is acknowledged.

${ }^{\dagger}$ CEREMADE, Université Paris IX Dauphine Place du Maréchal de Lattre de Tassigny 75775 Paris Cedex 16, France (cmouhot@ceremade.dauphine.fr).
} 
cross-section $\Sigma\left(v-v_{*}, \sigma\right)$ by the formula $\left.B=\left|v-v_{*}\right| \Sigma\right)$. On physical grounds, it is assumed that $B \geq 0$ and $B=B\left(\left|v-v_{*}\right|, \cos \theta\right)$ is a function of $\left|v-v_{*}\right|$ and $\cos \theta$, where $\theta \in[0, \pi]$ is the deviation angle between $v^{\prime}-v_{*}^{\prime}$ and $v-v_{*}$, defined by

$$
\cos \theta=\frac{v-v_{*}}{\left|v-v_{*}\right|} \cdot \sigma
$$

Boltzmann's collision operator has the fundamental properties of conserving mass, momentum and energy

$$
\int_{\mathbb{R}^{N}} Q(f, f) \phi(v) d v=0, \quad \phi(v)=1, v,|v|^{2}
$$

and satisfying Boltzmann's $H$ theorem, which can be formally written as

$$
\mathcal{D}(f):=-\frac{d}{d t} \int_{\mathbb{R}^{N}} f \log f d v=-\int_{\mathbb{R}^{N}} Q(f, f) \log (f) d v \geq 0 .
$$

The $H$ functional $H(f)=\int f \log f$ is the opposite of the entropy of the solution. Boltzmann's $H$ theorem implies that any equilibrium distribution function has the form of a Maxwellian distribution

$$
M(\rho, u, T)(v)=\frac{\rho}{(2 \pi T)^{N / 2}} \exp \left(-\frac{|u-v|^{2}}{2 T}\right),
$$

where $\rho, u, T$ are the density, mean velocity and temperature of the gas

$$
\rho=\int_{\mathbb{R}^{N}} f(v) d v, \quad u=\frac{1}{\rho} \int_{\mathbb{R}^{N}} v f(v) d v, \quad T=\frac{1}{N \rho} \int_{\mathbb{R}^{N}}|u-v|^{2} f(v) d v,
$$

which are determined by the mass, momentum and energy of the initial datum thanks to the conservation properties. As a result of the process of entropy production pushing towards local equilibrium combined with the constraints of conservation laws, solutions are thus expected to converge to a unique Maxwellian equilibrium. Up to a normalization we set without restriction $M(v)=e^{-|v|^{2}}$ as the Maxwellian equilibrium, or equivalently $\rho=\pi^{N / 2}, u=0$ and $T=1 / 2$.

1.2. Motivation. The relaxation to equilibrium is studied since the works of Boltzmann and it is at the core of the kinetic theory. The motivation is to provide an analytic basis for the second principle of thermodynamics for a statistical physics model of a gas out of equilibrium. Indeed Boltzmann's famous $H$ theorem gives an analytic meaning to the entropy production process and identifies possible equilibrium states. In this context, proving convergence towards equilibrium is a fundamental step to justify Boltzmann model, but cannot be fully satisfactory as long as it remains based on non-constructive arguments. Indeed, as suggested implicitly by Boltzmann when answering critics of his theory based on Poincaré recurrence Theorem, the validity of the Boltzmann equation breaks for very large time (see [35, Chapter 1, Section 2.5] for a discussion). It is therefore crucial to obtain quantitative informations on the time scale of the convergence, in order to show that this time scale is much smaller than the time scale of validity of the model. Moreover, constructive arguments often provide new qualitative insights into the model, for instance, here they give a better understanding of the dependency of the rate of convergence according to the collision kernel and the initial datum. 
1.3. Assumptions on the collision kernel. The main physical case of application of this paper is that of hard spheres in dimension $N=3$, where (up to a normalization constant) $B\left(\left|v-v_{*}\right|, \cos \theta\right)=\left|v-v_{*}\right|$. More generally we shall make the following decoupling assumption on the collision kernel: we assume that $B$ takes the product form

$$
B\left(\left|v-v_{*}\right|, \cos \theta\right)=\Phi\left(\left|v-v_{*}\right|\right) b(\cos \theta),
$$

where $\Phi$ and $b$ are nonnegative functions not identically equal to 0 . This decoupling assumption is made for the sake of simplicity and could probably be relaxed at the price of technical complications. Additional assumptions on the collision kernel shall be given in each section. Let us recall, nevertheless, the fundamental class of inverse power-law interaction models, for which $\Phi(z)=C_{\Phi}|z|^{\gamma}$ for some $\gamma \in(-N, 1]$ and $C_{\Phi}>$ 0 , and $\theta \mapsto b(\cos \theta)$ is not integrable at $\theta \sim 0$. The classical vocabulary denotes by hard potentials the case $\gamma>0$, Maxwell molecules the case $\gamma=0$, and soft potentials the case $\gamma<0$. Grad's angular cutoff assumption (see [21]) means a truncation so that $\theta \mapsto b(\cos \theta)$ is integrable on $[0, \pi]$.

In the case where $B$ is locally integrable, we can define the so-called collision frequency

$$
\nu(v)=\int_{\mathbb{R}^{N} \times \mathbb{S}^{N-1}} \Phi\left(\left|v-v_{*}\right|\right) b(\cos \theta) M\left(v_{*}\right) d v_{*} d \sigma .
$$

We denote by $\nu_{0} \geq 0$ the minimum value of $\nu$. It is easily seen that for inverse powerlaw collision kernels, this minimum value is positive as soon as $\gamma \geq 0$.

1.4. Linearization. We define the linearized collision operator $\mathcal{L}$ by

$$
\mathcal{L}(g)=Q(M+g, M+g)-Q(g, g)=Q(M, g)+Q(g, M) .
$$

We shall consider this operator on several functional spaces. The classical spectral theory of the Boltzmann collision operator, as pioneered by Hilbert [23], is done on $g \in L^{2}\left(M^{-1}\right)$. However the Cauchy theory for the nonlinear spatially homogeneous Boltzmann equation corresponds to the functional space $g \in L^{1}\left(1+|v|^{2}\right)$. We shall therefore introduce intermediate functional spaces in order to connect these two theories in the last section.

Let us recall some spectral theory's notions. Let us consider a linear unbounded operator $T: \mathcal{B} \rightarrow \mathcal{B}$ on the Banach space $\mathcal{B}$, defined on a dense domain $\operatorname{Dom}(T) \subset \mathcal{B}$. Then we adopt the following notations and definitions:

- we denote by $N(T) \subset \mathcal{B}$ the null space of $T$;

- $T$ is said to be closed if its graph is closed in $\mathcal{B} \times \mathcal{B}$.

In the following definitions, $T$ is assumed to be closed:

- the resolvent set of $T$ denotes the set of complex numbers $\xi$ such that $T-\xi$ is bijective from $\operatorname{Dom}(T)$ to $\mathcal{B}$ and the inverse linear operator $(T-\xi)^{-1}$, defined on $\mathcal{B}$, is bounded (see [24, Chapter 3, Section 5]);

- we denote by $\Sigma(T) \subset \mathbb{C}$ the spectrum of $T$, that is the complementary set of the resolvent set of $T$ in $\mathbb{C}$;

- an eigenvalue is a complex number $\xi \in \mathbb{C}$ such that $N(T-\xi)$ is not reduced to $\{0\}$;

- we denote $\Sigma_{d}(T) \subset \Sigma(T)$ the discrete spectrum of $T$, i.e., the set of discrete eigenvalues, that is the eigenvalues isolated in the spectrum and with finite multiplicity (i.e., such that the spectral projection associated with this eigenvalue has finite dimension, see [24, Chapter 3, Section 6]); 
- for a given discrete eigenvalue $\xi$, we shall call the eigenspace of $\xi$ the range of the spectral projection associated with $\xi$

- we denote $\Sigma_{e}(T) \subset \Sigma(T)$ the essential spectrum of $T$ defined by $\Sigma_{e}(T)=\Sigma(T) \backslash$ $\Sigma_{d}(T)$

- when $\Sigma(T) \subset \mathbb{R}_{-}$, we say that $T$ has a spectral gap when the distance between 0 and $\Sigma(T) \backslash\{0\}$ is positive, and the spectral gap denotes this distance;

- the operator $T$ is said to be sectorial when $\Sigma(T) \subset\{|\arg (\xi-\lambda)| \geq \pi / 2+w\}$ for some $w>0$ and some $\lambda \in \mathbb{R}$, and its resolvent satisfies the control: for any $\varepsilon>0$, there is $M_{\varepsilon}>0$ such that $\left\|(T-\xi)^{-1}\right\| \leq M_{\varepsilon} /|\xi-\lambda|$ for $\xi \in\{|\arg (\xi-\lambda)| \leq$ $\pi / 2+w-\varepsilon\}$ (see [24, Chapter 9, Section 1]).

1.5. Notation. In the sequel we shall denote $\langle\cdot\rangle=\sqrt{1+|\cdot|^{2}}$. For any Borel function $w: \mathbb{R}^{N} \rightarrow \mathbb{R}_{+}$, we define the weighted Lebesgue space $L^{p}(w)$ on $\mathbb{R}^{N}$ $(p \in[1,+\infty])$, by the norm

$$
\|f\|_{L^{p}(w)}=\left[\int_{\mathbb{R}^{N}}|f(v)|^{p} w(v) d v\right]^{1 / p}
$$

if $p<+\infty$ and

$$
\|f\|_{L^{\infty}(w)}=\sup _{v \in \mathbb{R}^{N}}|f(v)| w(v)
$$

when $p=+\infty$. The weighted Sobolev spaces $W^{k, p}(w)(p \in[1,+\infty]$ and $k \in \mathbb{N})$ are defined by the norm

$$
\|f\|_{W^{k, p}(w)}=\left[\sum_{|s| \leq k}\left\|\partial^{s} f\right\|_{L^{p}(w)}^{p}\right]^{1 / p}
$$

with the notation $H^{k}(w)=W^{k, 2}(w)$.

\section{The classical linearized theory, from Hilbert to Grad}

It is well-known from the classical theory of the linearized operator (see [22] or [19, Chapter 7, Section 1]) that the operator $\mathcal{L}$ on $L^{2}\left(M^{-1}\right)$ with domain $L^{2}\left(\nu^{2} M^{-1}\right)$ is closed and self-adjoint, and satisfies

$$
\begin{aligned}
\langle g, \mathcal{L} g\rangle_{L^{2}\left(M^{-1}\right)=} \int_{\mathbb{R}^{N}} g(\mathcal{L} g) M^{-1} d v & =-\frac{1}{4} \int_{\mathbb{R}^{N} \times \mathbb{R}^{N} \times \mathbb{S}^{N-1}} \Phi\left(\left|v-v_{*}\right|\right) b(\cos \theta) \\
& {\left[\left(\frac{g}{M}\right)_{*}^{\prime}+\left(\frac{g}{M}\right)^{\prime}-\left(\frac{g}{M}\right)_{*}-\left(\frac{g}{M}\right)\right]^{2} M M_{*} d v d v_{*} d \sigma \leq 0 . }
\end{aligned}
$$

This implies that its spectrum is included in $\mathbb{R}_{-}$. Its null space is

$$
N_{L^{2}\left(M^{-1}\right)}(\mathcal{L})=\operatorname{Span}\left\{M, v_{1} M, \ldots, v_{N} M,|v|^{2} M\right\} .
$$

These two properties correspond to the linearization of Boltzmann's $H$ theorem.

Let us denote by $D(g)=-\langle g, \mathcal{L} g\rangle_{L^{2}\left(M^{-1}\right)}$ the Dirichlet form for $-\mathcal{L}$. The existence of a spectral gap $\lambda>0$ can be written as

$$
\forall g \in L^{2}\left(M^{-1}\right), g \perp N_{L^{2}\left(M^{-1}\right)}(\mathcal{L}), \quad D(g) \geq \lambda\|g\|_{L^{2}\left(M^{-1}\right)}^{2} .
$$


The first step in the study of the linearized collision operator $\mathcal{L}$ was done by Hilbert (on the hard spheres model), who introduced a decomposition $\mathcal{L}=\mathcal{K}-\nu$ of $\mathcal{L}$ between a non-local part $\mathcal{K}$ (compact in $L^{2}\left(M^{-1}\right)$ ) and a multiplication part by the collision frequency $\nu$. This result was used by Hilbert for the construction of the so-called Hilbert expansion via the Fredholm alternative. This decomposition was then used by Carleman [12] to show the existence of a spectral gap for the hard spheres model. Then Grad [22] generalized this decomposition to hard potentials with angular cutoff or Maxwell molecules with angular cutoff, and obtained the existence of a spectral gap for these interactions. The proof relied on Weyl's Theorem for selfadjoint operators, which asserts that the essential spectrum is stable under (relatively) compact perturbation. Hence the essential spectrum of $\mathcal{L}$ is given by the one of $-\nu$ (which lies a positive distance away from 0 when $\gamma \geq 0$ ). Since the operator is nonpositive, it has only discrete eigenvalues in $\left(-\nu_{0}, 0\right]$, possibly accumulating only at $-\nu_{0}$.

It was also already observed that the Dirichlet form is monotonous according to $B$, which enables to prove the existence of a spectral gap for any collision kernel $B$ controlled from below by some cutoff hard potentials collision kernel. Moreover as discussed in [18, Chapter 4, Section 6], it was proved in [25] that $\mathcal{L}$ has an infinite number of discrete negative eigenvalues in the interval $\left(-\nu_{0}, 0\right)$, which implies that the spectral gap $\lambda$ satisfies $0<\lambda<\nu_{0}$. In fact the proof in [25] was done for hard spheres, but the argument applies to any cutoff hard potential collision kernel as well (see [18, Chapter 4, Section 6]).

Before going into the next section, let us note that this classical theory of the linearized collision operator made an important breakthrough: it showed exponential convergence in some linearized settings, and motivated subsequent development of the perturbative theory of the Boltzmann equation, see [33] for instance where the first smooth global solutions for the spatially inhomogeneous Boltzmann equation were constructed. However, its main drawback was that it was unable to provide any information on the size of the spectral gap.

\section{Explicit spectral gap estimates}

At about the same time as the work of Grad, another theory appears in the study of the Boltzmann equation. In the particular case of Maxwell molecules $(\gamma=0)$, it has been obtained (see $[37,38]$ ) a complete and explicit study of the spectrum of $\mathcal{L}$ in $L^{2}\left(M^{-1}\right)$, by symmetry arguments. Indeed, in the particular case where the collision kernel is independent on the modulus of the relative velocity, the collision operator lends itself to an extensive Fourier transform analysis, as first noticed by Bobylev, see $[6]$.

However, whenever $\Phi$ depends on the modulus of the relative velocity and the collision frequency is bounded from below by some positive number (for instance for the important physical case of hard spheres), the spectral gap was shown to exist by Grad's argument, but no quantitative information was provided, in particular on its size. In the work [4], we propose a new method to show the existence of a spectral gap for any hard potentials (in the generalized sense (3.1)), which is geometrical and based on a physical argument. It gives explicit estimates and deals with the whole operator, with or without angular cutoff.

Let us write down the assumptions for the collision kernel $B$ (in addition to the decoupling assumption (1.2)):

- The kinetic part $\Phi$ is bounded from below at infinity, i.e.,

$$
\exists R \geq 0, c_{\Phi}>0 \quad \mid \forall r \geq R, \Phi(r) \geq c_{\Phi} .
$$


This assumption holds for hard potentials (and hard spheres).

- The angular part $b$ satisfies

$$
c_{b}=\inf _{\sigma_{1}, \sigma_{2} \in \mathbb{S}^{N-1}} \int_{\sigma_{3} \in \mathbb{S}^{N-1}} \min \left\{b\left(\sigma_{1} \cdot \sigma_{3}\right), b\left(\sigma_{2} \cdot \sigma_{3}\right)\right\} d \sigma_{3}>0 .
$$

This assumption covers all the physical cases.

The main theorem of this work is

Theorem 3.1. Under the assumptions (1.2), (3.1), (3.2), the Dirichlet form D (in $L^{2}\left(M^{-1}\right)$ ) of the linearized collision operator with collision kernel $B=\Phi b$ satisfies

$$
\forall g \in L^{2}\left(M^{-1}\right), g \perp N_{L^{2}\left(M^{-1}\right)}(\mathcal{L}), \quad D(g) \geq C_{\Phi, b} D_{0}(g),
$$

where $D_{0}(g)$ stands for the Dirichlet form (in $L^{2}\left(M^{-1}\right)$ ) of the linearized collision operator with collision kernel $B_{0} \equiv 1$ and

$$
C_{\Phi, b}=\left(\frac{c_{\Phi} c_{b} e^{-4 R^{2}}}{32\left|\mathbb{S}^{N-1}\right|}\right)
$$

with $R, c_{\Phi}, c_{b}$ being defined in (3.1), (3.2).

As a consequence we deduce that

$$
\forall g \in L^{2}\left(M^{-1}\right), g \perp N_{L^{2}\left(M^{-1}\right)}(\mathcal{L}), \quad D(g) \geq C_{\Phi, b}\left|\lambda_{0}\right|\|g\|_{L^{2}\left(M^{-1}\right)}^{2} .
$$

Here $\lambda_{0}$ is the spectral gap (that is the modulus of the first non-zero eigenvalue) in $L^{2}\left(M^{-1}\right)$ of the linearized Boltzmann operator with $B_{0} \equiv 1$, which equals in dimension 3 (for density $\pi^{3 / 2}$, momentum 0 and temperature $1 / 2$, see [6])

$$
\lambda_{0}=\pi\left(\frac{\pi}{2}\right)^{3 / 2} \int_{0}^{\pi} \sin ^{3} \theta d \theta=\left(\frac{\pi}{2}\right)^{3 / 2} \frac{4 \pi}{3} .
$$

REMARK 3.2. Let us mention that in the same work, similar results are derived for the linearized Landau operator for hard potentials by grazing collision limit.

As an application of Theorem 3.1, let us give explicit controls for the spectral gap $\lambda=\lambda_{b, \gamma}(\rho, u, T)$ of the Boltzmann linearized operator with $b \geq 1$ and $\Phi(z)=|z|^{\gamma}$, $\gamma>0$, in dimension 3 , and for a steady state with density $\rho \geq 0$, momentum $u \in \mathbb{R}^{3}$, and temperature $T>0$. By scaling and translation arguments, it is straightforward that

$$
\lambda_{b, \gamma}(\rho, u, T)=\rho T^{\left(\frac{N+\gamma}{2}\right)} \lambda_{b, \gamma}(1,0,1) .
$$

Then $c_{b} \geq\left|\mathbb{S}^{2}\right|$ and for any given $R$ we can take $c_{\Phi}=R^{\gamma}$. Thus we get

$$
\lambda_{b, \gamma}(1,0,1) \geq 2^{\gamma / 2}\left(\frac{R^{\gamma} e^{-4 R^{2}}}{32}\right) \frac{4 \pi}{3}
$$

for any $R>0$ (the factor $2^{\gamma / 2}$ comes from the fact that $\lambda_{0}$ was computed for density $\pi^{3 / 2}$, momentum 0 and temperature $1 / 2$ ). An easy computation leads to the lower bound

$$
\lambda_{b, \gamma}(1,0,1) \geq 2^{\gamma / 2} \frac{\pi(\gamma / 8)^{\gamma / 2} e^{-\gamma / 2}}{24}
$$


by optimizing the free parameter $R$. For instance, in the hard spheres case $(b=1$ and $\gamma=1)$, we obtain a lower bound $\lambda_{1,1}(1,0,1) \geq c \approx 0.04$.

The idea of the proof is to work directly on the Dirichlet form (without requiring to some decomposition of the collision operator and perturbative theory anymore) and to reduce the case of hard potentials (in the generalized sense (3.1)) to the Maxwellian case. The difficulty is to deal with the cancellations of the collision kernel, and most importantly those of the kinetic part $\Phi$ on the diagonal $v=v_{*}$.

A first ingredient is the following inequality (which is a corollary of [17, Theorem 2.4])

$$
\begin{aligned}
\int_{\mathbb{R}^{N}} \int_{\mathbb{R}^{N}}|\varphi(x)-\varphi(y)|^{2}|x-y|^{\gamma} & M(x) M(y) d x d y \\
& \geq K_{\gamma} \int_{\mathbb{R}^{N}} \int_{\mathbb{R}^{N}}|\varphi(x)-\varphi(y)|^{2} M(x) M(y) d x d y
\end{aligned}
$$

for $\gamma \geq 0, \varphi$ some function, and

$$
K_{\gamma}=\frac{1}{4 \int_{\mathbb{R}^{N}} M} \inf _{x, y \in \mathbb{R}^{N}} \int_{\mathbb{R}^{N}} \min \left\{|x-z|^{\gamma},|z-y|^{\gamma}\right\} M(z) d z .
$$

The proof of (3.3) relies strongly on the existence of a "triangle inequality" for the function $F(x, y)=|\varphi(x)-\varphi(y)|^{2}$ integrated, which follows immediately here from

$$
F(x, y) \leq 2 F(x, z)+2 F(z, y) .
$$

All the point is thus to adopt suitable representations of the linearized collision operator, which contain some integrals of this form, and to estimate these integrals using some "triangular inequality" for the integrated function. Cancellations for $b$ are dealt with by considering the integral according to the variables $x=\left(v-v_{*}\right) /\left|v-v_{*}\right|$ and $y=\left(v^{\prime}-v_{*}^{\prime}\right) /\left|v^{\prime}-v_{*}^{\prime}\right|$ on the sphere (see Figure 3.1 where the intermediate variable is $\left.z=\left(w-w_{*}\right) /\left|w-w_{*}\right|\right)$. Cancellations of $\Phi$ are dealt with by considering the integration according to the variables $x=v \cdot\left(v^{\prime}-v\right) /\left|v^{\prime}-v\right|$ and $y=v^{\prime} \cdot\left(v^{\prime}-v\right) /\left|v^{\prime}-v\right|$ on $\mathbb{R}$ (see Figure 3.2 where the intermediate variable is $z=u \cdot\left(v^{\prime}-v\right) /\left|v^{\prime}-v\right|$ ).

Physically this corresponds to replacing in the Dirichlet form the collisions which cancel the collision kernel by some sequences of collisions for which the collision kernel is controlled from below. More precisely, for instance, to treat collisions which cancel $\Phi$ (small relative velocity), we replace each collision which has a small relative velocity by a sequence (two indeed) of intermediate collisions with large relative velocities in order to use (3.1) (see Figure 3.2).

\section{Explicit coercivity estimates}

In this section, we shall present the results of the work [28], which generalize the explicit spectral gap estimates into explicit coercivity estimates for any inverse power-law interactions.

We assume that the collision kernel $B$ satisfies (1.2) and:

- The kinetic part $\Phi$ is bounded from below by a power-law:

$$
\forall r \geq 0, \quad \Phi(r) \geq C_{\Phi} r^{\gamma}
$$

where $\gamma \in(-N, 1]$ and $C_{\Phi}>0$ is some constant. Collision kernels deriving from interaction potentials behaving like inverse-power laws satisfy this assumption, as well as hard spheres collision kernels. 


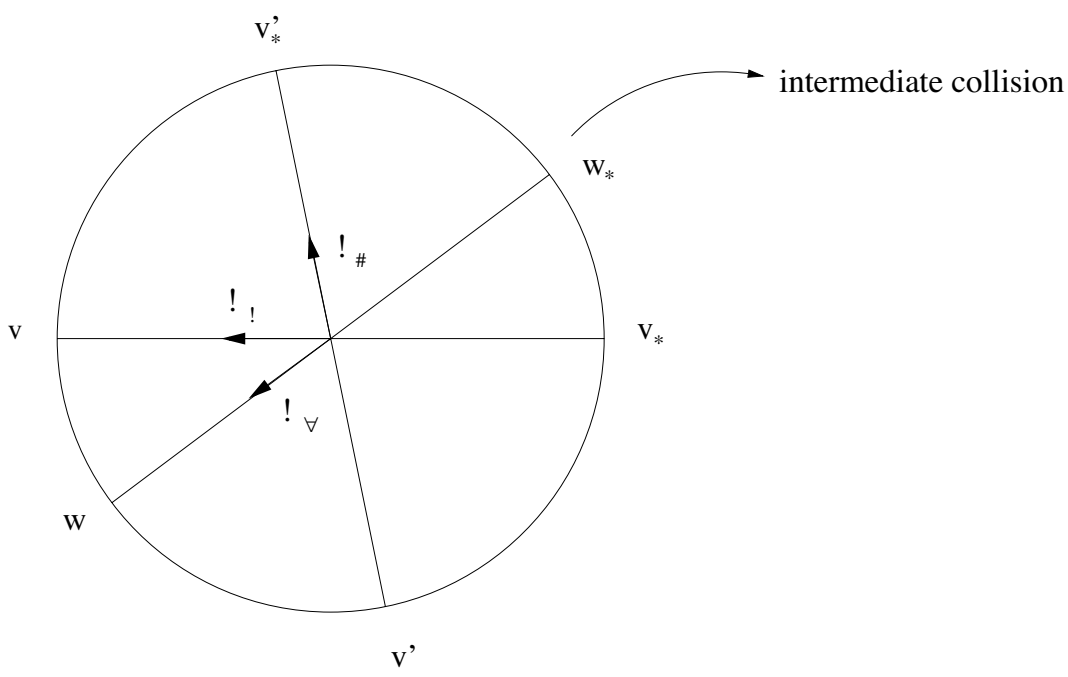

FIG. 3.1. Introduction of an intermediate collision to treat cancellations of $b$

- The angular part $b$ satisfies (3.2). Moreover, in order to obtain regularity estimates when the collision kernel is not locally integrable, we shall assume the more accurate control from below

$$
\forall \theta \in(0, \pi], \quad b(\cos \theta) \geq \frac{c_{b}}{\theta^{N-1+\alpha}}
$$

for some constant $c_{b}>0$ and $\alpha \in[0,2)$ (note that assumption (4.2) implies straightforwardly assumption (3.2)). The goal of this control is to measure the strength of the angular singularity, which is related to the regularity properties of the collision operator (as already noticed in [1] for instance).

It was proved in [10] that the linearized collision operator for soft potentials $(\gamma<0)$ with cutoff has no spectral gap. But if one allows a loss on the algebraic weight of the norm, it was proved in [20] a "degenerated spectral gap" result of the form:

$$
\forall g \in L^{2}\left(M^{-1}\right), g \perp N_{L^{2}\left(M^{-1}\right)}(\mathcal{L}), \quad D(g) \geq C\left\|g\langle v\rangle^{\gamma / 2}\right\|_{L^{2}\left(M^{-1}\right)}^{2}
$$

where $\gamma<0$ is the exponent in (4.1). The proof was based on inequalities proved in [10] together with Weyl's Theorem and it gave no informations on $C$.

In the work [28], we extend and complete the works [20] and [4] by

- giving a constructive proof of estimate (4.3) for soft potentials (with or without cutoff);

- extending it to hard potentials $(\gamma>0$ ) (note that for hard potentials this estimate is stronger than the usual spectral gap estimate);

- giving a coercivity result in local Sobolev spaces for the linearized Boltzmann operator with a non locally integrable collision kernel, and discussing the consequence on its spectrum. 


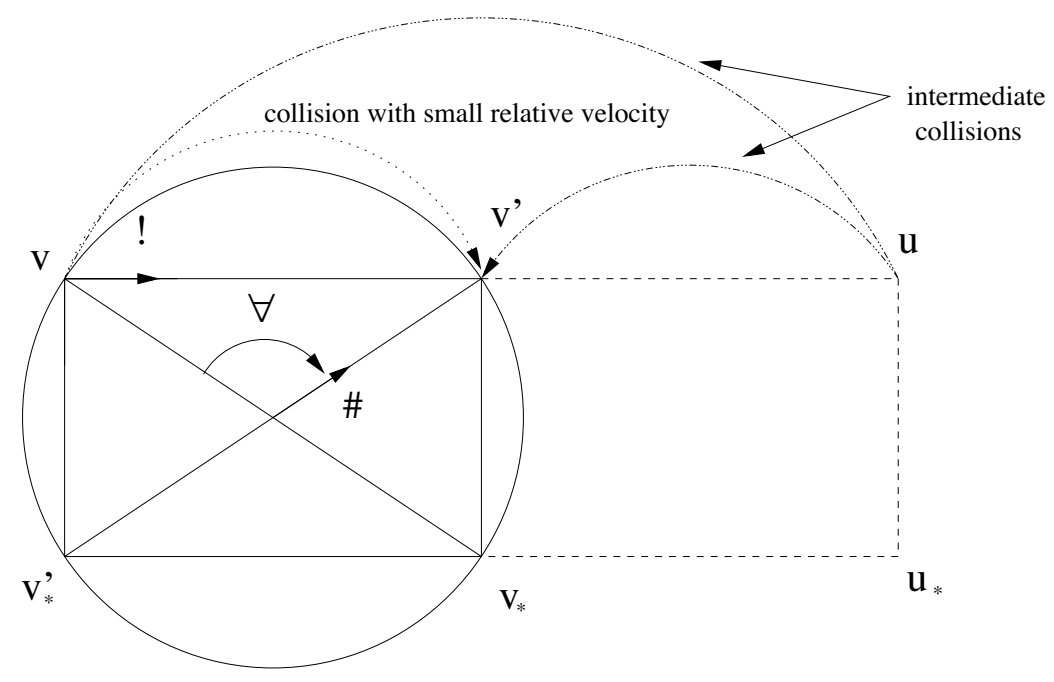

FIG. 3.2. Introduction of an intermediate collision to treat cancellations of $\Phi(z)$ at $z \sim 0$

In the following theorem, $H_{\mathrm{loc}}^{\alpha / 2}$ denotes the space of functions whose restriction to any compact set $K$ of $\mathbb{R}^{N}$ belongs to $H^{\alpha / 2}(K)=\left\{h \in L^{2}(K)\right.$ s. t. $(1-\Delta)^{-\alpha / 4} h \in$ $\left.L^{2}(K)\right\}$ (here $L^{2}(K)$ denotes space of functions square integrable on $K$ ).

The main result of this work is:

THEOREM 4.1. Under the assumptions (1.2), (4.1), (3.2), the linearized Boltzmann operator $\mathcal{L}$ with collision kernel $B=\Phi b$ satisfies

$$
\forall g \in L^{2}\left(M^{-1}\right), g \perp N_{L^{2}\left(M^{-1}\right)}(\mathcal{L}), \quad D(g) \geq C_{\gamma}\left\|g\langle v\rangle^{\gamma / 2}\right\|_{L^{2}\left(M^{-1}\right)}^{2}
$$

where $C_{\gamma}$ is an explicit constant depending only on $\gamma, C_{\Phi}, c_{b}$, and the dimension $N$.

When moreover the collision kernel is not locally integrable and b satisfies (4.2), $\mathcal{L}$ satisfies (4.4) and

$$
\forall g \in L^{2}\left(M^{-1}\right), g \perp N_{L^{2}\left(M^{-1}\right)}(\mathcal{L}), \quad D(g) \geq C_{\gamma, \alpha}\|g\|_{H_{\text {loc }}^{\alpha / 2}}^{2}
$$

where $C_{\gamma, \alpha}$ is an explicit constant depending only on $\gamma, \alpha, C_{\Phi}, C_{b}, c_{b}$ and the dimension $N$.

\section{REMARK 4.2.}

1. When the collision kernel is locally integrable, the collision frequency $\nu$ is finite, and the estimate (4.4) can be written in the following form:

$$
\forall g \in L^{2}\left(M^{-1}\right), g \perp N_{L^{2}\left(M^{-1}\right)}(\mathcal{L}), \quad D(g) \geq \bar{C}\|g \nu\|_{L^{2}\left(M^{-1}\right)}^{2}
$$

for some explicit constant $\bar{C}>0$. 
2. When the collision kernel is not locally integrable and $b$ satisfies (4.2), a natural conjecture would be that the estimate (4.5) improves into:

$$
\forall g \in L^{2}\left(M^{-1}\right), g \perp N_{L^{2}\left(M^{-1}\right)}(\mathcal{L}), \quad D(g) \geq C_{\gamma, \alpha}\left\|g\langle v\rangle^{\gamma / 2}\right\|_{H^{\alpha / 2}\left(M^{-1}\right)}^{2} .
$$

We were not able to obtain this coercivity estimate, however we give its consequence in terms of local regularity.

3. When $\gamma>0$ and $\alpha>0$, one can easily deduce from Theorem 4.1 that the operator $\mathcal{L}$ has compact resolvent (see [28]), which implies that its spectrum is purely discrete in this case (note that it is also true when $\gamma=0$ and $\alpha \geq 0$ by explicit diagonalization).

4. Similar results have been derived in the same paper [28] for the linearized Landau collision operator. We also mention the forthcoming work [30] where some new spectral gap estimates improving on (4.4) in the non-integrable case are obtained.

In the case of hard potentials, the idea of the proof is to decompose the operator into a part satisfying the desired coercivity estimate and a bounded part, and use the spectral gap estimates. This argument is reminiscent of an argument of Grad [22, Section 5] used to study the decrease of the eivenvectors of the linearized Boltzmann operator for hard potentials, and it was already noticed in [5] for instance. Nevertheless, it is the first time that it is used to obtain explicit estimates (thanks to the results in [4] presented in the previous section).

For soft potentials we use a dyadic decomposition of the Dirichlet form depending on the modulus of the relative velocity. Combined with technical estimates on the non-local part of the linearized collision operators and the spectral gap estimates from the Maxwell case, it enables to reconstruct a lower bound with the appropriate weight.

Finally, the proof of the coercivity estimates in local Sobolev spaces for the linearized Boltzmann operator with a non-locally integrable collision kernel is inspired by the previous works $[26,34,1]$ on the regularizing properties of the nonlinear collision operator and by the structure of the linearized Landau operator in the grazing collision limit. The main task is to identity a "diffusive part", which shares coercivity properties similar to the one of a fractional Laplacian, and a remaining bounded part.

5. Applications to the quantitative study of rate of convergence: connection to the nonlinear theory

We present in this section the work [29], which is devoted to the study of the asymptotic behavior of solutions to the spatially homogeneous Boltzmann equation for hard potentials with cutoff. On the one hand it was proved by Arkeryd [3] by nonconstructive arguments that spatially homogeneous solutions (with finite mass and energy) of the Boltzmann equation for hard spheres converge towards equilibrium with exponential rate, with no information on the rate of convergence and the constants (in fact, the proof in this paper required some moment assumptions, but the latter can be relaxed with the results about appearance and propagation of moments, as can be found in [40]). On the other hand it was proved in [31] a quantitative convergence result with rate $O\left(t^{-\infty}\right)$ for these solutions.

In the work [29] we improve and fill the gap between these results by

- showing exponential convergence towards equilibrium by constructive arguments (with explicit rate and constants); 
- showing that the spectrum of the linearized collision operator in the narrow space $L^{2}\left(M^{-1}\right)$ dictates the asymptotic behavior of the solution in a much more general setting, as was conjectured in [16] on the basis of the study of the Maxwell case.

We assume that we deal with hard spheres collision kernel:

$$
B\left(\left|v-v_{*}\right|, \cos \theta\right)=C_{B}\left|v-v_{*}\right|
$$

for some $C_{B}>0$. The results of [29] apply more generally to hard potentials with cutoff collision kernels (under some suitable technical assumptions). Let us recall that under this assumption on $B$, it has been shown in $[2,27]$ that for any initial datum $0 \leq f_{0} \in$ $L^{1}\left(1+|v|^{2}\right)$, there is a unique global nonnegative solution in $L^{1}\left(1+|v|^{2}\right)$ preserving mass, momentum and energy.

Let us review briefly existing results in the study of convergence to equilibrium for the spatially homogeneous Boltzmann equation for hard spheres. On the basis of the $H$ theorem and suitable a priori estimates, various authors gave results of $L^{1}$ convergence to equilibrium by compactness arguments for the spatially homogeneous Boltzmann equation with hard potentials and angular cutoff (for instance Carleman [11], Arkeryd [2], etc.). These results provide no information at all on the rate of convergence. Then, on the basis of these compactness results and the classical linearized theory presented in Section 2, Arkeryd gave in [3] the first (non-constructive) proof of exponential convergence in $L^{1}$ for the spatially homogeneous Boltzmann equation with hard potentials and angular cutoff. His result was generalized to $L^{p}$ spaces $(1 \leq p<+\infty)$ by Wennberg [39].

At the beginning of the nineties, several difficulties still had to be overcome in order to get a quantitative result of exponential convergence:

(i) No estimate was available for the spectral gap in $L^{2}\left(M^{-1}\right)$ for hard spheres.

(ii) There is no known a priori estimate for the nonlinear problem showing propagation (let alone appearance) of the norm $L^{2}\left(M^{-1}\right)$. Matching results obtained in this space and the physical space $L^{1}\left(1+|v|^{2}\right)$ is one of the main difficulties, and was treated in [3] by a non-constructive argument.

(iii) Finally, any estimate deduced from a linearization argument is valid only in a neighborhood of the equilibrium, and the use of compactness arguments to deduce that the solution enters this neighborhood (as e.g. in [3]) would prevent any hope of obtaining explicit estimate.

REMARK 5.1. Note that in the Maxwell case with cutoff, all these difficulties have been solved. We have already mentioned that in this case, Wang-Chang and Uhlenbeck [37, 38 ] and then Bobylev [6] were able to obtain a complete and explicit diagonalization of the linearized collision operator in $L^{2}\left(M^{-1}\right)$. Then specific metric well suited to the collision operator for Maxwell molecules allowed the achievement of the goals sketched at the beginning of this section (under additional assumptions on the initial datum), see [16] and [15]. However, it seems that the proofs are strongly restricted to the Maxwellian case.

In order to solve the point (iii), quantitative estimates in the large have been developed since the beginning of the nineties, directly on the nonlinear equation, by relating the entropy production functional to the relative entropy: see $[13,14,32$, $36,31]$. The latter paper states, for hard spheres, quantitative convergence towards equilibrium with rate $O\left(t^{-\infty}\right)$ for solutions in $L^{1}\left(1+|v|^{2}\right)$. Unfortunately, it was proved in [8] that Cercignani's conjecture on entropy production is false in the case 
of hard potentials, even for smooth solutions with strong algebraic decay (although "almost true", in the sense of [36]). It means that one cannot establish in this setting a linear inequality relating the entropy production functional and the relative entropy, which would yield exponential convergence directly on the nonlinear equation.

Point (i) was solved in [4] (see Section 3).

In order to solve the remaining obstacle of point (ii), the strategy of [29] is to prove explicit linearized estimates of convergence to equilibrium in the space $L^{1}\left(\exp \left(a|v|^{s}\right)\right)$ with $a>0$ and $0<s<\gamma / 2$, on which we have explicit results of appearance and propagation of the norm for solution of (1.1), and thus which can be connected to the quantitative nonlinear results in [31]. It leads to study the linearized operator $\mathcal{L}$ in the space $L^{1}\left(\exp \left(a|v|^{s}\right)\right)$, which has no hilbertian self-adjointness structure.

The main result of this work is:

THEOREM 5.2. Let $B$ be a collision kernel satisfying assumption (5.1). Let $\lambda \in\left(0, \nu_{0}\right)$ be the spectral gap of the linearized operator $\mathcal{L}$ in $L^{2}\left(M^{-1}\right)$. Let $f_{0}$ be a nonnegative initial datum in $L^{1}\left(1+|v|^{2}\right)$. Then the solution $f=f(t, v)$ to the spatially homogeneous Boltzmann equation (1.1) with initial datum $f_{0}$ satisfies: for any $0<\mu \leq \lambda$, there is a constant $C$, which depends explicitly on $B$, the mass and energy of $f_{0}$, on $\mu$ and on a lower bound on $\nu_{0}-\mu$, such that

$$
\|f(t, \cdot)-M\|_{L^{1}\left(\mathbb{R}^{N}\right)} \leq C e^{-\mu t} .
$$

REMARK 5.3.

Let us recall that from Section 3 (work [4]), one deduces (in dimension 3 for $C_{B}=1$ ) that $\lambda$ is controlled from below by some $c \approx 0.04$. Hence by choosing $\mu=c$ we get a completely explicit rate of exponential convergence, since

$$
\nu_{0}=\left|\mathbb{S}^{2}\right| \int_{\mathbb{R}^{3}} M(v)|v| d v
$$

is explicit.

We also state the functional analysis result used in the proof of Theorem 5.2 and which has interest in itself. We consider $\overline{\mathcal{L}}$, that is the unbounded linearized collision operator on the space $L^{1}\left(\exp \left(a|v|^{s}\right)\right.$ ) with $a>0$ and $0<s<\gamma / 2$ (with domain $\left.\operatorname{Dom}(\overline{\mathcal{L}})=L^{1}\left(\nu \exp \left(a|v|^{s}\right)\right)\right)$, and $\mathcal{L}$, that is the unbounded self-adjoint linearized collision operator on $L^{2}\left(M^{-1}\right)$ (with domain $L^{2}\left(\nu^{2} M^{-1}\right)$ ). These operators are closed and we have

THEOREM 5.4. Let $B$ be a collision kernel satisfying assumption (5.1). Then the spectrum $\Sigma(\overline{\mathcal{L}})$ of $\overline{\mathcal{L}}$ is equal to the spectrum $\Sigma(\mathcal{L})$ of $\mathcal{L}$. Moreover $\overline{\mathcal{L}}$ and $\mathcal{L}$ have the same eigenvectors associated to discrete eigenvalues.

REMARK 5.5.

1. This theorem essentially means that enlarging the functional space from $g \in L^{2}\left(M^{-1}\right)$ to $g \in L^{1}\left(\exp \left(a|v|^{s}\right)\right)$ does not yield new eigenvectors for the linearized collision operator.

2. It implies in particular that $\overline{\mathcal{L}}$ only has non-degenerate eigenspaces associated with its discrete eigenvalues, since this is true for the self-adjoint operator $\mathcal{L}$. This is related to the fact that the optimal convergence rate $\mu=\lambda$ is reached. Note that it 
seems to be the first time that such a result is obtained, since both the quantitative study in [16] for Maxwell molecules and the non-constructive results of [3] for hard spheres only prove a convergence like $O\left(e^{-\mu t}\right)$ for any $\mu<\lambda$, where $\lambda$ is the corresponding spectral gap. It also yields a simple form of the first term in the asymptotic expansion (see [29]).

Let us explain shortly the method of the proof. The main idea is to establish quantitative estimates of exponential decay on the evolution semigroup of $\overline{\mathcal{L}}$. They are used to estimate the rate of convergence when the solution is close to equilibrium (where the linear part of the collision operator is dominant), whereas the existing nonlinear entropy method, combined with some a priori estimates in $L^{1}\left(\exp \left(a|v|^{s}\right)\right)$, is used to estimate the time necessary to enter a given neighborhood of equilibrium,

Concerning the first step, that is, to show the exponential decay on the semigroup of $\overline{\mathcal{L}}$, it is done by proving that $\overline{\mathcal{L}}$ is sectorial, with explicit control on the resolvent, and with the same spectrum as $\mathcal{L}$ (see the introduction for the definition of a sectorial operator). This relies essentially on Hilbert's decomposition of $\overline{\mathcal{L}}$ into a local part (the multiplication by the collision frequency) and a non-local part, and then on the proof that the non-local part is well approximated by some truncated operator mapping functions of $L^{1}\left(\exp \left(a|v|^{s}\right)\right)$ into $L^{2}\left(M^{-1}\right)$. From this one can deduce that the resolvent of $\overline{\mathcal{L}}$ at $\xi \in \mathbb{C}$ exists as soon as the one of $\mathcal{L}$ exists, with an explicit relation between their norms. This shows that $\Sigma(\overline{\mathcal{L}}) \subset \Sigma(\mathcal{L})$, and yields the desired sectoriality estimate on $\overline{\mathcal{L}}$. More accurate results on the essential spectrum of $\overline{\mathcal{L}}$ (in order to obtain Theorem 5.4) can be obtained using perturbative arguments as Grad $\operatorname{did}$ for $\mathcal{L}$

Concerning the second step, that is, the connection to the nonlinear theory, it is mainly based on three ingredients: a Gronwall argument to deal with the remaining quadratic term, some sharp versions of Povzner inequalities (in the spirit of $[7,9]$ ) to show the appearance and propagation of the $L^{1}\left(\exp \left(a|v|^{s}\right)\right)$ norm, and the quantitative result of convergence to equilibrium of [31] to obtain an explicit time for the solution to the nonlinear Boltzmann equation to enter into some given neighborhood of equilibrium.

\section{REFERENCES}

[1] R. Alexandre, L. Desvillettes, C. Villani and B. Wennberg, Entropy dissipation and long-range interactions, Arch. Rat. Mech. Anal., 152, 327-355, 2000.

[2] L. Arkeryd, On the Boltzmann equation, Arch. Rat. Mech. Anal., 45, 1-34, 1972.

[3] L. Arkeryd, Stability in $L^{1}$ for the spatially homogeneous Boltzmann equation, Arch. Rat. Mech. Anal., 103, 151-167, 1988.

[4] C. Baranger and C. Mouhot, Explicit spectral gap estimates for the linearized Boltzmann and Landau operators with hard potentials, Rev. Mat. Iberoam., 21, 819-841, 2005.

[5] C. Bardos, R. E. Caflisch and B. Nicolaenko, The Milne and Kramers problems for the Boltzmann equation of a hard sphere gas, Comm. Pure Appl. Math., 39, 323-352, 1986.

[6] A. V. Bobylev, The theory of the nonlinear spatially uniform Boltzmann equation for Maxwell molecules, Mathematical physics reviews, Soviet Sci. Rev. Sect. C Math. Phys. Rev., 7, 111-233, 1988.

[7] A. V. Bobylev, Moment inequalities for the Boltzmann equation and applications to spatially homogeneous problems, J. Stat. Phys., 88, 1183-1214, 1997.

[8] A. V. Bobylev and C. Cercignani, On the rate of entropy production for the Boltzmann equation, J. Stat. Phys., 94, 603-618, 1999.

[9] A. V. Bobylev, I. M. Gamba and V. A. Panferov, Moment inequalities and high-energy tails for the Boltzmann equations with inelastic interactions, J. Stat. Phys., 116, 1651-1682, 2004.

[10] R. E. Caflisch, The Boltzmann equation with a soft potential. I. Linear, spatially-homogeneous, Comm. Math. Phys., 74, 71-95, 1980. 
[11] T. Carleman, Sur le théorie de l'équation intégrodifférentielle de Boltzmann, Acta Math., 60, 369-424, 1932.

[12] T. Carleman, Problèmes mathématiques dans la théorie cinétique des gaz, Publ. Sci. Inst. Mittag-Leffler., Uppsala, 1957.

[13] E. A. Carlen and M. C. Carvalho, Strict entropy production bounds and stability of the rate of convergence to equilibrium for the Boltzmann equation, J. Stat. Phys., 67, 575-608, 1992.

[14] E. A. Carlen and M. C. Carvalho, Entropy production estimates for Boltzmann equations with physically realistic collision kernels, J. Stat. Phys., 74, 743-782, 1994.

[15] E. A. Carlen and X. Lu, Fast and slow convergence to equilibrium for Maxwellian molecules via Wild sums, J. Stat. Phys., 112, 59-134, 2003.

[16] E. A. Carlen, E. Gabetta and G. Toscani, Propagation of smoothness and the rate of exponential convergence to equilibrium for a spatially homogeneous Maxwellian gas, Comm. Math. Phys., 199, 521-546, 1999.

[17] J. A. Carrillo, R. J. McCann and C. Villani, Kinetic equilibration rates for granular media and related equations: entropy dissipation and mass transportation estimates, Rev. Mat. Iberoamericana, 19(3), 971-1018, 2003.

[18] C. Cercignani, The Boltzmann Equation and its Applications, Appl. Math. Sci., SpringerVerlag, New York, 67, 1988.

[19] C. Cercignani, R. Illner and M. Pulvirenti, The Mathematical Theory of Dilute Gases, SpringerVerlag, New York, 1994.

[20] F. Golse and F. Poupaud, Un résultat de compacité pour l'équation de Boltzmann avec potentiel mou. Application au problème de demi-espace, C. R. Acad. Sci. Paris Sér. I Math., 303, 585-586, 1986.

[21] H. Grad, Principles of the kinetic theory of gases, in Flügge's Handbuch des Physik, SpringerVerlag, XII, 205-294, 1958.

[22] H. Grad, Asymptotic theory of the Boltzmann equation. II., Rarefied Gas Dynamics (Proc. 3rd Internat. Sympos., Palais de l'UNESCO, Paris, 1962), I, 26-59, 1963.

[23] D. Hilbert, Grundzüge einer Allgemeinen Theorie der Linearen Integralgleichungen, Math. Ann., 72, 1912. Chelsea Publ., New York, (1953).

[24] T. Kato, Perturbation Theory for Linear Operators, Springer-Verlag, Berlin, 1995.

[25] I. Kuščer, M. M. R. Williams, Phys. Fluids, 10, 1922-1927, 1967.

[26] P.-L. Lions, Regularity and compactness for Boltzmann collision operators without angular cut-off, C. R. Acad. Sci. Paris Sér. I Math., 326, 37-41, 1998.

[27] S. Mischler and B. Wennberg, On the spatially homogeneous Boltzmann equation, Ann. Inst. H. Poincar Anal. Non Linaire, 16, 467-501, 1999.

[28] C. Mouhot, Explicit coercivity estimates for the linearized Boltzmann and Landau operators, Comm. Part. Diff. Equations, 31, 1321-1348, 2006.

[29] C. Mouhot, Rate of convergence to equilibrium for the spatially homogeneous Boltzmannn equation, Comm. Math. Phys., 261, 629-672, 2006.

[30] C. Mouhot and R. Strain, Explicit spectral gap and coercivity estimates for linearized Boltzmann collision operators without angular cutoff, Math.AP/0607495, preprint.

[31] C. Mouhot and C. Villani, Regularity theory for the spatially homogeneous Boltzmann equation with cut-off, Arch. Rat. Mech. Anal., 173, 169-212, 2004.

[32] G. Toscani and C. Villani, Sharp entropy dissipation bounds and explicit rate of trend to equilibrium for the spatially homogeneous Boltzmann equation, Comm. Math. Phys., 203, 667-706, 1999.

[33] S. Ukai, On the existence of global solutions of a mixed problem for the nonlinear Boltzmann equation, Proc. Japan Acad., 50, 179-184, 1974.

[34] C. Villani, Regularity estimates via the entropy dissipation for the spatially homogeneous Boltzmann equation without cut-off, Rev. Mat. Iberoam., 15, 335-352, 1999.

[35] C. Villani, A review of mathematical topics in collisional kinetic theory, Handbook of mathematical fluid dynamics, North-Holland, Amsterdam, I, 71-305, 2002.

[36] C. Villani, Cercignani's conjecture is sometimes true and always almost true, Comm. Math. Phys., 243, 455-490, 2003.

[37] C. S. Wang Chang and G. E. Uhlenbeck, On the Propagation of Sound in Monoatomic Gases, Univ. of Michigan Press, Ann Arbor, Michigan (quoted in [22]).

[38] C. S. Wang Chang, G. E. Uhlenbeck and J. Boer, Studies in Statistical Mechanics, NorthHolland, Amsterdam, Vol. V., 1970.

[39] B. Wennberg, Stability and exponential convergence in $L^{p}$ for the spatially homogeneous Boltzmann equation, Nonlinear Anal., 20, 935-964, 1993.

[40] B. Wennberg, Entropy dissipation and moment production for the Boltzmann equation, J. Stat. Phys., 86, 1053-1066, 1997. 